\title{
The psychological impact of SARS: a matter of heart and mind
}

\author{
Kang Sim, Hong Choon Chua
}

ß See related article page 793

$\mathrm{S}$ ARS was the first novel infectious disease to emerge in the 21 st century. Its dramatic appearance in major cities around the world, together with the fact that $20 \%$ of the 8400 infected individuals were health care workers, ${ }^{1}$ prompted epidemiologists and other scientists to move swiftly to study the disease and identify its causal agent. ${ }^{2,3}$ We now know that SARS is associated with a previously unrecognized virus, SARS-CoV. ${ }^{4}$ Rapid diagnostic tests using the polymerase chain reaction are being developed, ${ }^{5}$ and the treatment regimens used in the outbreaks of 2002-2003 continue to be reviewed and evaluated. ${ }^{6}$

It is interesting that, even during the height of the outbreaks, researchers were also trying to understand and measure the psychosocial effects of SARS. ${ }^{7-9}$ Compared with the available literature on the biology of infectious diseases, there have been considerably fewer published reports on the psychosocial impact of SARS and other disease outbreaks. Thus, the article in this issue by Nickell and colleagues $^{10}$ (see page 793) makes an important contribution toward a better understanding of this often neglected area.

Their study was carried out in a large teaching hospital in Toronto in April 2003, during the peak of the first phase of the SARS outbreak in the city. The authors found significant levels of psychiatric morbidity, in that almost twothirds of the staff surveyed reported increased levels of concern for personal and family health, and almost one-third of a subset of respondents who completed a 12-item General Health Questionnaire had scores indicating emotional distress. Their findings are consistent with those of studies on SARS in Taiwan, where up to $75 \%$ of health care workers experienced psychiatric morbidity (Dr. Mian-Yoon Chong, Chang Gung Memorial Hospital, Kaohsiung, Taiwan: personal communication, 2004). In Singapore, 238 cases of SARS were diagnosed during the outbreak, and we found that psychiatric morbidity was also present in up to $21 \%$ of health care workers within a large, primary health care setting (unpublished data).

Maunder and colleagues ${ }^{7}$ reported that some health care workers at Toronto's Mount Sinai Hospital experienced intense emotional reactions during the SARS outbreak, including the fear of contagion, feelings of stigmatization, loneliness, boredom, anger, anxiety and a sense of uncertainty. Similar reactions have been described in health care workers in the context of a previous botulism outbreak. ${ }^{11}$
These psychological responses may be associated with certain health-seeking behaviours. For example, Leung and colleagues ${ }^{9}$ found that individuals in their community sample who had greater risk perception as well as moderate anxiety were more likely than those without to take precautions against SARS.

Although these studies of the psychosocial impact of SARS are both important and commendable, more work is needed. The psychological well-being of health care workers who deal with disease outbreaks, the responses of the public and the outcomes of intervention programs need to be set as priority areas for research, as the report of Canada's National Advisory Committee on SARS and Public Health urges. ${ }^{12}$ Whether or not SARS outbreaks recur, ${ }^{13}$ there will be other new emerging pathogens. The intriguing and worrisome characteristic of an emerging infectious disease is that the precise cause is at first unknown. This uncertainty in itself may increase the level of psychosocial morbidity. ${ }^{14,15}$

Three elements are needed in future psychosocial research on the impact of infectious disease. First, we need to adopt a systemic perspective. Just as it is important to appreciate and evaluate the psychosocial impact of any emerging infectious disease or nosocomial infection on patients and health care workers, it is equally important to determine the psychological effects of the disease on often unseen populations such as family members, nonmedical staff, medical colleagues in the community and the general public. This will enable a more comprehensive and balanced planning of efforts to alleviate such psychosocial burden or to mitigate its onset in the future. Second, because the psychological impact may persist or evolve over time, prospective research is warranted. Longitudinal studies will allow an assessment of the important determinants of psychological distress as well as the protective effects of certain coping strategies, with potential application in early identification of cases requiring more intervention. Third, in this era of evidencebased medicine, the outcomes of psychosocial interventions should be evaluated. This includes assessment of both individual- and group-based interventions and of other measures such as staff educational sessions, public education and the responsive communication of new information updates. A clearer understanding of these beneficial or therapeutic elements will facilitate the implementation of strategic mental health responses for people at risk. 
Emerging new infections such as SARS exert a significant psychological impact on health care workers and the community at large, which in some instances requires flexible and appropriate interventions. It is an area that urgently needs more research. Enhancing the psychological wellbeing of health care providers, their families and the community is a crucial tool in the continuing vigilance and fight against emerging infectious diseases.

The authors are with the Department of Adult Psychiatry III, Institute of Mental Health / Woodbridge Hospital, Singapore.

Competing interests: None declared.

Contributors: Both authors contributed substantially to the drafting and revising of this article.

\section{References}

1. World Health Organization. Summary table of SARS cases by country, 1 November 2002-7 August 2003. Geneva: The Organization; 2003 Aug 15. Available: www.who.int/csr/sars/country/2003_08_15/en (accessed 2004 Feb 6).

2. Poutanen SM, Low DE, Henry B, Finkelstein S, Rose D, Green K, et al. Identification of severe acute respiratory syndrome in Canada. $N$ Engl $7 \mathrm{Med}$ 2003;348(20):1995-2005.

3. Lee N, Hui D, Wu A, Chan P, Cameron P, Joynt GM, et al. A major outbreak of severe acute respiratory syndrome in Hong Kong. $N$ Engl $7 \mathrm{Med}$ 2003;348(20):1986-94

4. Drosten C, Gunther S, Preiser W, van der Werf S, Brodt HR, Becker S, et al. Identification of a novel coronavirus in patients with severe acute respiratory syndrome. N Engl 7 Med 2003;348:1967-76.
5. Tang P, Louie M, Richardson SE, Smieja M, Simor AE, Jamieson F, et al. Interpretation of diagnostic laboratory tests for severe acute respiratory syndrome: the Toronto experience. CMA7 2004;170(1):47-54

6. Zhaori G. Antiviral treatment of SARS: Can we draw any conclusions? [editorial]. CMA7 2003;169(11):1165-6.

7. Maunder R, Hunter J, Vincent L, Bennett J, Peladeau N, Leszcz M, et al. The immediate psychological and occupational impact of the 2003 SARS outbreak in a teaching hospital. CMA7 2003;168(10):1245-51.

8. Avendano M, Derkach P, Swan S. Clinical course and management of SARS in health care workers in Toronto: a case series. CMA7 2003;168(13):1649-60.

9. Leung GM, Lam TH, Ho LM, Ho SY, Chan BH, Wong IO, et al. The impact of community psychological responses on outbreak control for severe acute respiratory syndrome in Hong Kong. 7 Epidemiol Community Health 2003;57(11):857-63.

10. Nickell LA, Crighton EJ, Tracy CS, Al-Enazy H, Bolaji Y, Hanjrah S, et al Psychosocial effects of SARS on hospital staff: survey of a large tertiary care institution. CMA7 2004;170(5):793-8.

11. Cohen RE, Anderson DL. Botulism: emotional impact on patient and family. 7 Psychosom Res 1986;30(3):321-6.

12. National Advisory Committee on SARS and Public Health. Learning from SARS: renewal of public bealth in Canada. Ottawa: Health Canada; Oct 2003 Available: www.hc-sc.gc.ca/english/protection/warnings/sars/learning.htm (accessed 2004 Feb 6).

13. Low DE. Why SARS will not return: a polemic [editorial]. CMAf 2004; 170(1):68-9.

14. Poole K, Hood K, Davis BD, Monypenny IJ, Sweetland H, Webster DJ, et al. Psychological distress associated with waiting for results of diagnostic investigations for breast disease. Breast 1999;8:334-8.

15. Thompson DR, Lopez V, Lee D, Twinn S. SARS: a perspective from a school of nursing in Hong Kong. 7 Clin Nurs 2004;13:131-5.

Correspondence to: Dr. Kang Sim, Department of Adult

Psychiatry III, Institute of Mental Health / Woodbridge Hospital,

10 Buangkok View, Singapore 539747; kang_sim@imh.com.sg
CMA CENTRE FOR

PHYSICIAN HEALTH AND WELL-BEING

HEALTHY PHYSICIANS, VIBRANT PROFESSION

\section{If you are feeling}

overwhelmed by

professional and personal

demands, you are not alone.

Research has found

that $45.7 \%$ of

Canadian physicians are

in an advanced

state of burnout.*
CMA believes in a culture of medicine that values the health of its practitioners and does not stigmatize those who seek personal assistance. CMA's new Centre for Physician Health and Well-being provides national leadership and advocacy on issues affecting the health and morale of Canadian physicians.

To learn more about the work of the centre or to access information and resources, visit the centre's new Web section at cma.ca or call 1877 CMA-4-YOU (1 877 262-4968).

The centre supports and complements the work of the provincial and territorial physician health programs but does not provide individual assessment, support or treatment advice. Contact numbers for the programs are available at cma.ca

*2003 CMA Physician Resource Questionnaire. 